\title{
Tratamientos Farmacológicos en Pacientes con Síndrome de Boca Urente: Revisión Sistemática
}

\author{
Pharmacologic Treatment of Burning Mouth Syndrome: A Systematic Review
}

Jared Linco Olave; Sebastián Riquelme Carrasco* \& Scarlette Hernández Vigueras**

\begin{abstract}
LINCO, O. J.; RIQUELME, C. S. \& HERNÁNDEZ, V. S. Tratamientos farmacológicos en pacientes con síndrome de boca urente: Revisión sistemática. Int. J. Odontostomat., 10(2):229-235, 2016.

RESUMEN: El objetivo fue determinar mediante una revisión sistemática, cuáles tratamientos farmacológicos para el Síndrome de Boca Urente (SBU) logran una reducción de síntomas, según Escala Visual Análoga (EVA). Se realizó una búsqueda bibliográfica en la bases de datos PubMed y SciELO, Trip Database, Scopus Database, EBSCO host y LILACS entre el 2005 y 2015. De 72 artículos, se seleccionaron un total de 11. Los tratamientos sistémicos usados fueron, Hipericum perforatum, Catuama, Clonazepam, Ácido alfa lipoico y Lafutidina. Entre los tratamientos tópicos, Aceite de oliva virgen enriquecido con licopeno, Lisozima lactoperoxidasa, Clonazepam y Capsaicina. Los fármacos que obtuvieron mejores resultados para el tratamiento del SBU fueron Lafutidina, Catuama, Clonazepam tópico y sistémico, y en menor grado Capsaicina.
\end{abstract}

PALABRAS CLAVE: síndrome de boca urente, tratamiento, fármacos sistémicos, fármacos tópicos, escala visual análoga, farmacoterapia.

\section{INTRODUCCIÓN}

El Síndrome de Boca Urente (SBU) es definido por la International Headache Society como "una sensación de ardor intraoral de la cual no hay una causa médica o dental que puede ser encontrada" (Headache Classification Subcommittee of the International Headache Society, 2004). Los síntomas se manifiestan con mayor frecuencia en los dos tercios anteriores de la lengua, paladar duro y labios (Evans \& Drage, 2005). La prevalencia varía de $0,7 \%$ a $15 \%$ en población general (Lipton et al., 1993; Tammiala-Salonen et al., 1993), afectando mayormente a mujeres, con una mayor prevalencia al aumentar de edad y después de la menopausia (Basker et al., 1978).

Su etiopatogenia es desconocida, siendo relacionado con posibles factores locales, sistémicos, o psicogénicos (Patton et al., 2007; Gremeau-Richard et al., 2004). El SBU a menudo es subdiagnosticado, y cuando se diagnostica tiene un mal manejo (Savage, 1996), ya que no hay pruebas o instrumentos de laboratorio específicos para establecer un diagnóstico de- finitivo (de Moraes et al., 2012), representando una condición clínica importante con síntomas agravantes, los cuales afectan directa o indirectamente la calidad de vida, lo que a menudo supone una carga reconocible en el paciente y en el sistema de atención de salud (Gurvits \& Tan, 2013).

Los tratamientos propuestos para mejorar los síntomas del SBU incluyen sialogogos, anestésicos tópicos en enjuague, ansiolíticos, antidepresivos, anticonvulsivos, compuestos herbales, ácido alfa lipoico, antihistamínicos, psicoterapia y acupuntura. La variedad de tratamientos actualmente en uso, demuestra que no hay evidencia de cómo debería ser manejado (Cano-Carrillo et al., 2014).

El objetivo de este estudio fue determinar a través de una revisión sistemática de ensayos clínicos, cuál o cuáles son los tratamientos farmacológicos que logran una disminución de la sintomatología según la Escala Visual Análoga (EVA) en pacientes con SBU.

\footnotetext{
"Escuela de Odontología, Facultad de Medicina, Universidad Austral de Chile, Valdivia, Chile.

* Instituto de Odontoestomatología, Facultad de Medicina, Universidad Austral de Chile, Valdivia, Chile.
} 


\section{MATERIAL Y MÉTODO}

Se realizó una revisión sistemática de la literatura en las bases de datos PubMed, SciELO, Trip Database, Scopus Database, EBSCO host y LILACS, el 24 de Julio del 2015. Los términos de búsqueda, filtros aplicados y resultados se encuentran en la Tabla I.

El flujograma de la selección de los artículos junto con los criterios de inclusión y exclusión aplicados se presenta en la Figura 1. Los artículos seleccionados fueron revisados de manera independiente por los autores, utilizando los criterios de selección descritos. Dos de los autores extrajeron los datos de interés necesarios, los cuales, fueron registrados en una tabla a través de Google Docs. Para evaluar la calidad de los ensayos clínicos seleccionados se utilizó la escala desarrollada y descrita por Jadad et al. (1996) cuya puntuación se presenta en la Tabla II.

\section{RESULTADOS}

En cuanto a las características metodológicas de los ensayos clínicos evaluados a través de la escala Jadad (Jadad et al.) solo dos artículos presentan calidad metodológica excelente (Cano-Carrillo et al., Cavalcanti \& da Silveira, 2009), siete artículos presentan calidad metodológica buena (Sardella et al., 2008; Spanemberg et al., 2012; Heckmann et al., 2012; Carbone et al., 2009; López-Jornet et al., 2009; Rodríguez de Rivera Campillo et al., 2010; Silvestre et al., 2012) y dos calidad metodológica aceptable (Marino et al., 2010; Toida et al., 2009).

Con respecto a las características y efecto de las intervenciones, de los 11 artículos seleccionados, los tratamientos sistémicos fueron hipericum perforatum (Sardella et al.) catuama (Spanemberg et al.), clonazepam (Heckmann et al.), ácido alfa

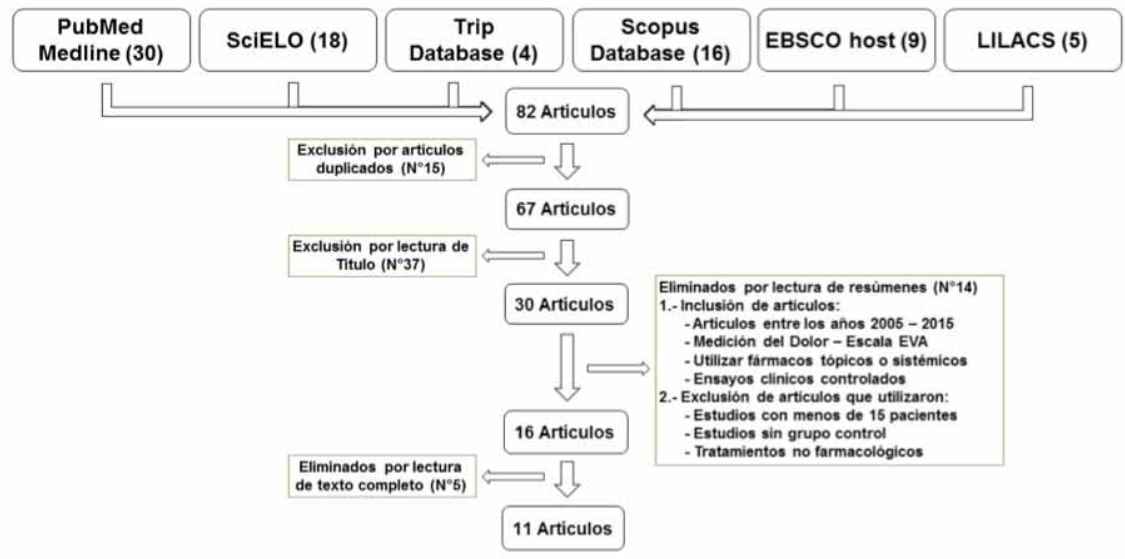

Fig. 1. Flujograma selección de artículos y criterios de selección.

Tabla I. Búsqueda realizada en las bases de datos, estrategia de búsqueda, filtros aplicados, número de artículos encontrados.

\begin{tabular}{|c|c|c|c|}
\hline Base de datos & Estrategia de Búsqueda & Límites de Búsqueda & n artículos \\
\hline PubMed/Medline & $\begin{array}{l}\text { "Burning Mouth Syndrome/therapy"[Mesh] AND } \\
\text { (Clinical Trial[ptyp] }\end{array}$ & $\begin{array}{l}\text { "2005/07/27"[PDat] : } \\
\text { "2015/07/24" } \\
\text { [PDat] "humans"[MeSH }\end{array}$ & 30 \\
\hline SciELO & $\begin{array}{l}\text { "Burning Mouth Syndrome" AND "Treatment" AND } \\
\text { "Pharmacotherapy" AND ("Systemic drug" OR } \\
\text { "Topical drug") }\end{array}$ & Yेear: $2005-2015$ & 18 \\
\hline Trip Database & $\begin{array}{l}\text { "(Title:Burning Mouth Syndrome)", "(Title: } \\
\text { Treatment)", “(Controlled Trial) }\end{array}$ & Year: $2005-2015$ & 4 \\
\hline Scopus Database & $\begin{array}{l}\text { "Burning Mouth Syndrome" AND "Clinical Trials" } \\
\text { AND "VAS". }\end{array}$ & Year: 2005 - 2015 & 16 \\
\hline EBSCO host & $\begin{array}{l}\text { "Burning Mouth Syndrome" AND "Treatment" AND } \\
\text { "Clinical Trials". }\end{array}$ & Year: 2005 - 2015 & 9 \\
\hline LILACS & $\begin{array}{l}\text { "Burning Mouth Syndrome" AND ("Treatment" OR } \\
\text { "Pharmacotherapy") AND "Systemic drug" OR } \\
\text { "Topical drug") }\end{array}$ & Year: 2005 - 2015 & 5 \\
\hline
\end{tabular}


LINCO, O. J.; RIQUELME, C. S. \& HERNÁNDEZ, V. S. Tratamientos farmacológicos en pacientes con síndrome de boca urente: Revisión sistemática. Int. J. Odontostomat., 10(2):229-235, 2016.

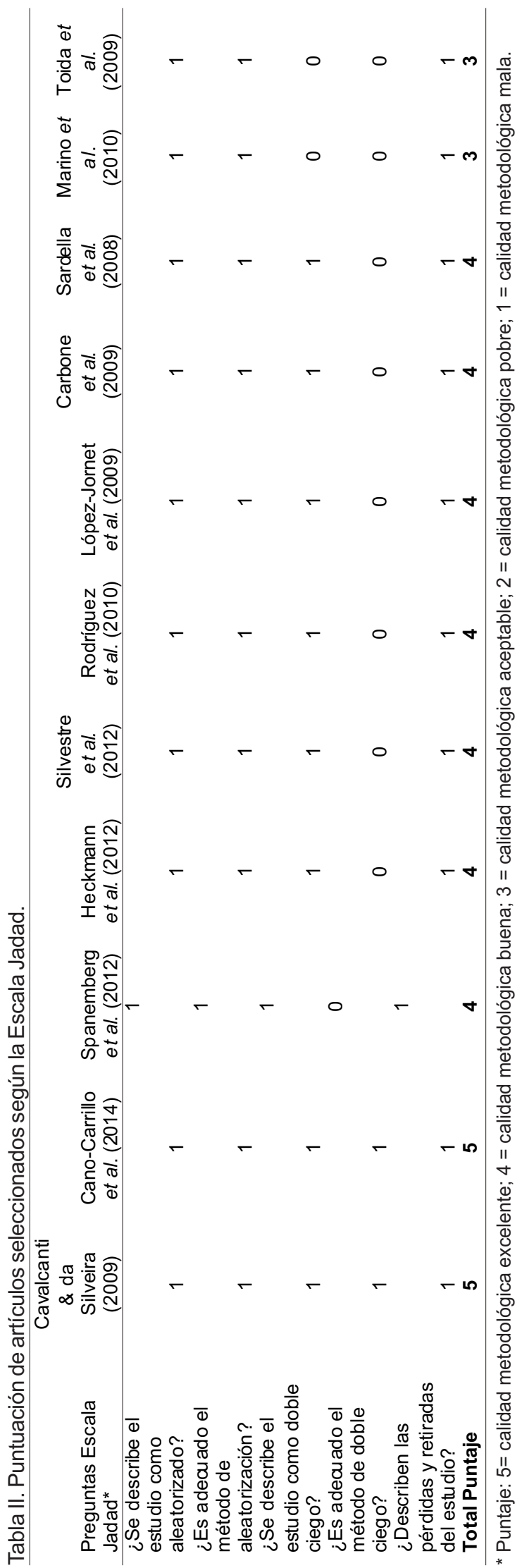

lipoico (ALA) (Calvacanti \& Silveira, Carbone et al., LópezJornet et al., Marino et al.) y lafutidina (Toida et al.). Los tratamientos tópicos fueron aceite de oliva virgen enriquecido con licopeno (Cano-Carrillo et al.), clonazepam tópico (Rodríguez de Rivera Campillo et al.), capsaicina (Silvestre et al.; Marino et al.) y lisozima lactoperoxidasa (Marino et al.). Las características y resultados de los estudios seleccionados se presentan en la Tabla III.

\section{DISCUSIÓN}

Fármacos Sistémicos. El extracto de Hipericum perforatum, es un derivado de una planta que ha sido usado en diferentes aplicaciones clínicas, comúnmente usado para el tratamiento de depresión, el cual no logró disminuir los síntomas en EVA con diferencia estadísticamente significativas respecto al grupo control (Sardella et al.). Catuama, utilizado ampliamente en Brasil, es un compuesto herbal a partir de 4 extractos de plantas medicinales, con propiedades analgésicas, antimicrobianas, antidepresivas y efecto vasodilatador. Su mecanismo de acción tiene efectos similares a los antidepresivos tricíclicos, demostrando eficacia en la recaptación de serotonina y dopamina. Logró demostrar una reducción estadísticamente significativa de síntomas en pacientes con SBU, representando una posible opción terapéutica (Spanemberg et al.).

El Clonazepam sistémico, es una benzodiacepina con efecto inhibidor del sistema nervioso central, usado como ansiolítico. Demostró una mayor reducción de síntomas en el grupo tratamiento, que en el control, siendo estadísticamente significativa. Tiene efectividad principalmente en bajas dosis, en pacientes más jóvenes y con cortos períodos de síntomas urentes (Heckmann et al.).

La Lafutidina, es un antagonista del receptor histamínico $\mathrm{H} 2$, la cual tiene un efecto sobre las neuronas aferentes sensibles a capsaicina (NASC), indicado para el tratamiento de úlceras gástricas. Fue administrado en conjunto con un enjuague de azuleno sulfonato en pacientes en tratamiento por gastritis y SBU, demostrando que puede reducir la intensidad urente, en un corto periodo de tratamiento, al ser comparado con un grupo control. Su mecanismo de acción sugiere que puede mejorar el flujo sanguíneo por mediación transitoria sobre el receptor potencial vaniloide tipo 1 (TRPV1), el cual está involucrado en la modulación y transmisión del dolor, aparte de que podría inhibir la acumulación de sustancia $\mathrm{P}$ y otros neuropéptidos asociados a la nocicepción (Toida et al.). Sin embargo, la muestra de pacientes de este estudio, estaba en tratamiento de gastritis, además de presentar SBU, factor que podría tener alguna influencia en los resultados. 


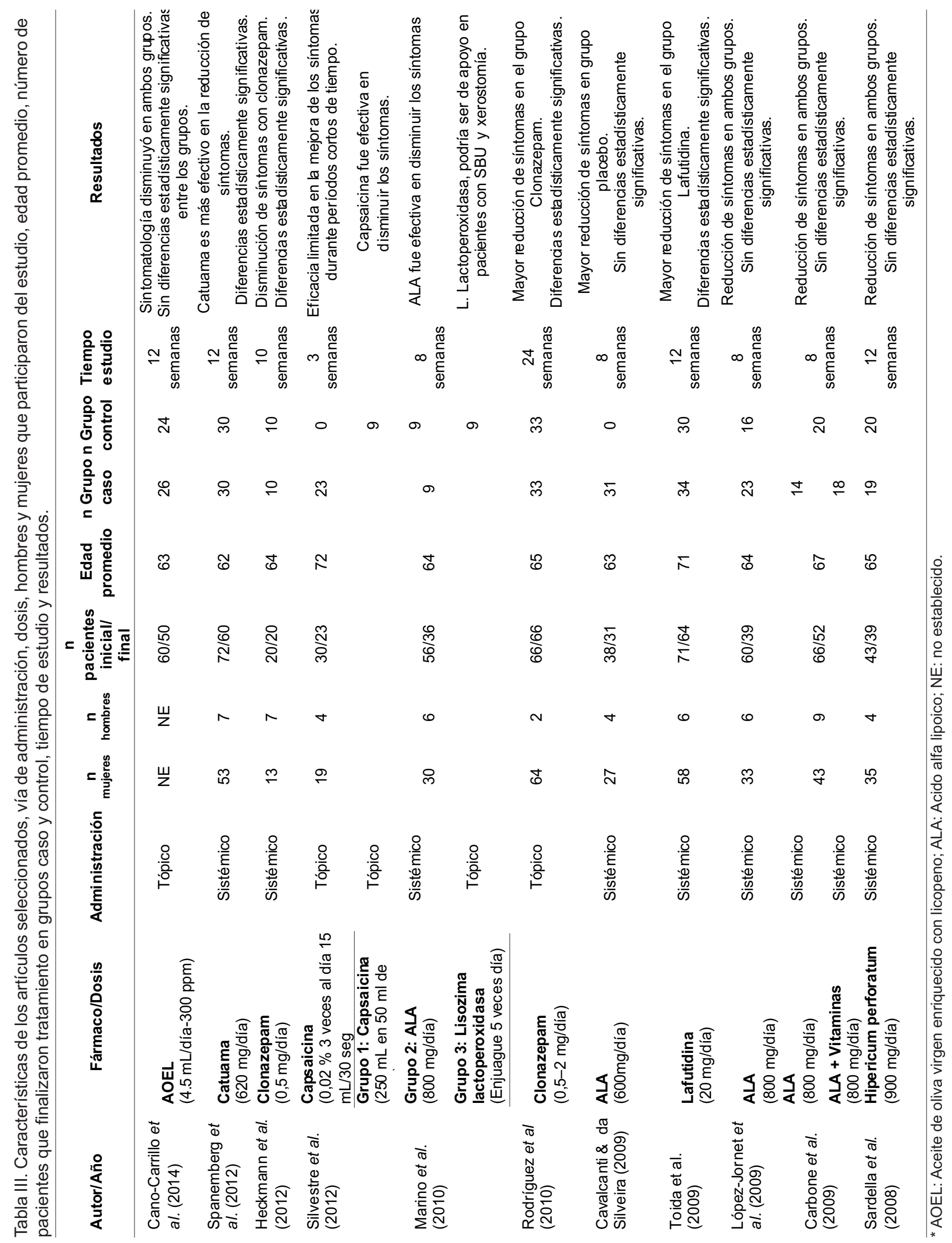


Ácido Alfa Lipoico (ALA), es un potente antioxidante con excelente biodisponibilidad, utilizado en la prevención y tratamiento de neuropatía diabética (Calvacanti \& da Silveira). Fue utilizado en cuatro estudios, siendo el tratamiento que presenta más evidencia publicada; sin embargo, en tres de los artículos no demostró eficacia significativa en disminuir los síntomas según EVA (Calvacanti \& da Silveira; Carbone et al.; López-Jornet et al.). Esto se contradice a estudios publicados anteriormente por Femiano et al. (2000, 2002), quien obtuvo resultados favorables logrando una mejoría del 76-97 \%. Carbone et al., expone que la evaluación de los síntomas se puede alterar por una relación interpersonal entre los pacientes y el investigador, al ser el mismo operador quien siguió el tratamiento de los pacientes. Calvacanti \& da Silveira, expone que el tamaño muestral y el corto periodo de tratamiento pudo variar sus resultados en comparación a los de Femiano et al. (2000, 2002). López-Jornet et al., explica que sus resultados pudieron variar, debido al extenso rango en la duración de la enfermedad entre los pacientes, ya que la cronicidad del dolor puede estar asociados con una peor respuesta al tratamiento, similar a lo descrito por Heckman et al., y Rodríguez de Rivera Campillo et al., en el tratamiento con Clonazepam. Al contrario, Marino et al., encontró una reducción estadísticamente significativa, respecto al grupo control con ALA, al igual que Femiano et al. $(2000,2002)$. Sin embargo, esta heterogeneidad de resultados, puede ser atribuida a las diferencias en la evaluación de los resultados entre los autores, o lo que se considera como significativo en la reducción de síntomas.

Tratamientos Tópicos. El aceite de oliva virgen enriquecido con licopeno, es un caroteno sin actividad de vitamina $A$, con actividad antioxidante, utilizado en enfermedades crónicas, el cual no demostró diferencias significativas en la disminución en EVA entre los grupos (Cano et al.). Clonazepam tópico, redujo en más del $50 \%$ los síntomas según EVA en el $76 \%$ del grupo tratamiento, siendo estadísticamente significativo en comparación al grupo control (Rodríguez de Rivera Campillo et al.). Es importante señalar que la reducción de síntomas fue más significativa en los pacientes que llevaban menor periodo de tiempo con SBU al igual que Heckmann et al. La administración tópica tiene una rápida actividad, lo que indicaría que actúa localmente mientras se disuelve en la boca, sin embargo, también puede tener absorción sublingual, dado que algunos pacientes relataron somnolencia; no obstante, cuando se administra oralmente, tiene efectos farmacológicos en 3-4 h (Rodríguez de Rivera Campillo et al.). Por lo tanto, clonazepam tópico al igual que sistémico repre- senta una opción válida, siendo más recomendada en pacientes con historia reciente de SBU.

La Capsaicina, utilizado comúnmente como tratamiento alternativo del dolor neuropático en general, tiene un mecanismo de acción relacionado con los receptores vaniloides en fibras nerviosas sensitivas tipo $C$, inhibiendo la síntesis y transporte axonal de sustancia P (Marino et al.; Silvestre et al.). Demostró una disminución de los síntomas, pero dentro de sus limitaciones está el efecto limitado en el tiempo, siendo necesario aplicar reiteradamente (Marino et al.). Sin embargo, en el estudio de Silvestre et al., algunos pacientes tuvieron aumento de los síntomas urentes después de la aplicación del enjuague, por lo tanto, recomienda su uso con algunas limitaciones, al contrario de Marino et al., quien no observó efectos adversos. Por último la Lisozima lactoperoxidasa, utilizada para el tratamiento de la xerostomía, controló con éxito los síntomas de los pacientes, pero es necesario de mayor investigación para ser confirmados estos resultados (Marino et al.).

Con respecto a los problemas metodológicos de los estudios, destacamos la baja prevalencia de SBU la cual no permite obtener grandes muestras, la variedad de criterios usados para clasificar a los pacientes según los síntomas y la ausencia de grupos controles en algunos estudios que no permiten cuantificar el real efecto de la intervención. Además, la diversidad de escalas usadas y las diferencias entre los estudios al determinar un efecto como significativo, generan confusión al analizar los efectos reales de los diferentes fármacos estudiados.

En una revisión, realizada por Zakrzewska et al. (2005), en la cual incluyeron 9 artículos a analizar, concluyeron que sólo tres tratamientos presentaron disminución de los síntomas en SBU, clonazepam, ALA y terapia cognitivo-conductual. Al contrario de sus resultados, con respecto a ALA, la mayoría de los estudios de esta revisión no obtuvieron resultados estadísticamente significativos (Calvacanti \& da Silveira; Carbone et al.; López-Jornet et al.), sin embargo, Zakrzewska et al., evitó dar una estimación general del efecto de ALA, debido a variaciones en los resultados y al hecho de que los pacientes de los ensayos de Femiano et al. $(2000,2002)$ podrían haberse solapado, por lo que la real efectividad de ALA es confuso. Los estudios que utilizaron Clonazepam en esta revisión, también presentan resultados favorables en el tratamiento de SBU (Heckmann et al., Rodríguez de Rivera Campillo et al.) 


\section{CONCLUSIÓN}

La diversidad de fármacos propuestos para el tratamiento sintomático de pacientes con SBU, señala la necesidad de establecer un tratamiento que demuestre una mayor eficacia en disminuir los síntomas. Catuama (Spanemberg et al.), Clonazepam tópico (Rodríguez de Rivera Campillo et al.), Clonazepam sistémico (Heckmann et al.) y Lafutidina (Toida et al.) tuvieron resultados con diferencias estadísticamente significativas según esta revisión y el uso de Capsaicina representan una opción terapéutica en disminuir los síntomas, pero con algunas limitaciones (Marino et al.; Silvestre et al.), mientras que los resultados con ALA son contradictorios (Calvacanti \& da Silveira; Carbone et al.; López-Jornet et al.; Marino et al.). Es necesario el desarrollo de nuevos ensayos clínicos aleatorizados, doble ciego, con grupo control, en condiciones controladas y por un mayor periodo de tiempo de estos fármacos que avalen sus resultados, utilizando idealmente EVA para poder homologar resultados y determinar la verdadera efectividad de la intervención.

\section{AGRADECIMIENTOS}

Esta investigación está basada en una tesis realizada como requerimiento para la obtención del grado académico de Licenciado en Odontología, de la Universidad Austral de Chile (Abril, 2016).

LINCO, O. J.; RIQUELME, C. S. \& HERNÁNDEZ, V. S. Pharmacologic treatment of burning mouth syndrome: A Systematic review. Int. J. Odontostomat., 10(2):229-235, 2016.

ABSTRACT: The aim of this study was to determine through a systematic review, which is the best drug treatment for burning mouth syndrome (SBU), measured on a Visual Analogue Scale. A scientific literature search was conducted in PubMed and SciELO, Trip Database, Database Scopus, EBSCO host and LILACS data between 2005 and 2015. Of a total of 72 articles, 11 were included for analysis. Systemic treatments were Lycopeneenriched virgin olive oil, Hypericum perforatum, Catuama, Clonazepam, Alpha lipoic acid; topical treatments were Lysozyme lactoperoxidase, Clonazepam, Capsaicin and Lafutidine. The best results obtained were with Lafutidine, Catuama, topical and systemic Clonazepam, and to a lesser degree Capsaicin.

KEY WORDS: burning mouth syndrome, treatment, systemic drug, topical drug, visual analogue scale, pharmacotherapy.

\section{REFERENCIAS BIBLIOGRÁFICAS}

Basker, R. M.; Sturdee, D. W. \& Davenport, J. C. Patients with burning mouths. A clinical investigation of causative factors, including the climacteric and diabetes. Br. Dent. J., 145(1):9-16, 1978.

Cano-Carrillo, P.; Pons-Fuster, A. \& López-Jornet, P. J. Efficacy of lycopene-enriched virgin olive oil for treating burning mouth syndrome: a double-blind randomised. J. Oral Rehabil., 41(4):296-305, 2014.

Carbone, M.; Pentenero, M.; Carrozzo, M.; Ippolito, A. \& Gandolfo, S. Lack of efficacy of alpha-lipoic acid in burning mouth syndrome: a double-blind, randomized, placebo-controlled study. Eur. J. Pain, 13(5):492-6, 2009.

Cavalcanti, D. R. \& da Silveira, F. R. Alpha lipoic acid in burning mouth syndrome--a randomized double-blind placebo-controlled trial. J. Oral Pathol. Med., 38(3):25461, 2009.

de Moraes, M.; do Amaral Bezerra, B. A.; da Rocha Neto, P. C.; de Oliveira Soares, A. C.; Pinto, L. P. \& de Lisboa Lopes Costa, A. Randomized trials for the treatment of burning mouth syndrome: an evidence-based review of the literature. J. Oral Pathol. Med., 41(4):281-7, 2012.

Evans, R. W. \& Drage, L. A. Burning mouth syndrome. Headache, 45(8):1079-81, 2005.

Femiano, F.; Gombos, F.; Scully, C.; Busciolano, M. \& De Luca, P. Burning mouth syndrome (BMS): controlled open trial of the efficacy of alpha-lipoic acid (thioctic acid) on symptomatology. Oral Dis., 6(5):274-7, 2000.

Femiano, F. \& Scully, C. Burning mouth syndrome (BMS): double blind controlled study of alpha-lipoic acid (thioctic acid) therapy. J. Oral Pathol. Med., 31(5):2679, 2002.

Gremeau-Richard, C.; Woda, A., Navez, M. L.; Attal, N.; Bouhassira, D.; Gagnieu, M. C.; Laluque, J. F.; Picard, P.; Pionchon, P. \& Tubert, S. Topical clonazepam in stomatodynia: a randomised placebo-controlled study. Pain, 108(1-2):51-7, 2004.

Gurvits, G. E. \& Tan, A. Burning mouth syndrome. World J. Gastroenterol., 19(5):665-72, 2013.

Headache Classification Subcommittee of the International Headache Society. The International Classification of Headache Disorders: 2nd edition. Cephalalgia, 24(Suppl. 1):9-160, 2004.

Heckmann, S. M.; Kirchner, E.; Grushka, M.; Wichmann, M. G. \& Hummel, T. A double-blind study on clonazepam in 
LINCO, O. J.; RIQUELME, C. S. \& HERNÁNDEZ, V. S. Tratamientos farmacológicos en pacientes con síndrome de boca urente: Revisión sistemática. Int. J. Odontostomat., 10(2):229-235, 2016.

patients with burning mouth syndrome. Laryngoscope, 122(4):813-6, 2012.

Jadad, A. R.; Moore, R. A.; Carroll, D.; Jenkinson, C.; Reynolds, D. J.; Gavaghan, D. J. \& McQuay, H. J. Assessing the quality of reports of randomized clinical trials: is blinding necessary? Control Clin. Trials, 17(1):112, 1996.

Lipton, J. A.; Ship, J. A. \& Larach-Robinson, D. Estimated prevalence and distribution of reported orofacial pain in the United States. J. Am. Dent. Assoc., 124(10):115-21, 1993.

López-Jornet, P.; Camacho-Alonso, F. \& Leon-Espinosa, S. Efficacy of alpha lipoic acid in burning mouth syndrome: a randomized, placebo-treatment study. J. Oral Rehabil., 36(1):52-7, 2009.

Marino, R.; Torretta, S.; Capaccio, P.; Pignataro, L. \& Spadari, F. Different therapeutic strategies for burning mouth syndrome: preliminary data. J. Oral Pathol. Med., 39(8):611-6, 2010.

Patton, L. L.; Siegel, M. A.; Benoliel, R. \& De Laat, A. Management of burning mouth syndrome: systematic review and management recommendations. Oral Surg. Oral Med. Oral Pathol. Oral Radiol. Endod., 103(Suppl.):S39-e1-13, 2007.

Rodríguez de Rivera Campillo, E.; López-López, J. \& Chimenos-Küstner, E. Response to topical clonazepam in patients with burning mouth syndrome: a clinical study. Bull. Group Int. Rech. Sci. Stomatol. Odontol., 49(1):1929, 2010.

Sardella, A.; Lodi, G.; Demarosi, F.; Tarozzi, M.; Canegallo, L. \& Carrassi, A. Hypericum perforatum extract in burning mouth syndrome: a randomized placebo-controlled study. J. Oral Pathol. Med., 37(7):395-401, 2008.

Savage, N. W. Burning mouth syndrome: patient management. Aust. Dent. J., 41(6):363-6, 1996.

Silvestre, F. J.; Silvestre-Rangil, J.; Tamarit-Santafé, C. \& Bautista, D. Application of a capsaicin rinse in the treatment of burning mouth syndrome. Med. Oral Patol. Oral Cir. Bucal, 17(1):e1-4, 2012.

Spanemberg, J. C.; Cherubini, K.; de Figueiredo, M. A.; Gomes, A. P.; Campos, M. M. \& Salum, F. G. Effect of an herbal compound for treatment of burning mouth syndrome: randomized, controlled, double-blind clinical trial. Oral Surg. Oral Med. Oral Pathol. Oral Radiol., 113(3):373-7, 2012.

Tammiala-Salonen, T.; Hiidenkari, K. \& Parvinen, T. Burning mouth in a Finnish adult population. Community Dent. Oral Epidemiol., 21(2):67-71, 1993.
Toida, M.; Kato, K.; Makita, H.; Long, N. K.; Takeda, T.; Hatakeyama, D.; Yamashita, T. \& Shibata, T. Palliative effect of lafutidine on oral burning sensation. J. Oral Pathol. Med., 38(3):262-8, 2009.

Zakrzweska, J. M.; Forssell, H. \& Glenny, A. M. Interventions for the treatment of burning mouth syndrome. Cochrane Database Syst. Rev., (1):CD002779, 2005.

Dirección para Correspondencia:

Scarlette Hernández Vigueras

Instituto de Odontoestomatología

Universidad Austral de Chile

Rudloff 1640

Valdivia

CHILE

Email: shernandez@uach.cl

Recibido : 17-03-2016

Aceptado: 14-07-2016 